\title{
affaissements miniers et exploitation partielle
}

\author{
par \\ J. Arcamone \\ M. Dejean \\ Ingénieurs au CERCHAR
}

Pour protéger les terrains de surface influencés par des exploitations minières souterraines, sans sacrifier délibérément les réserves, le mineur doit parfois envisager un dimensionnement des chantiers approprié s'il ne veut pas recourir à la solution très coûteuse du remblayage. C'est le cas, en particulier, quand l'exploitation se trouve placée sous des villes ou des installations industrielles.

En ménageant des piliers entre des bandes exploitées, on peut minimiser et parfois éviter les mouvements à la surface. Ces méthodes sont dites d'exploitation partielle par bandes et piliers. La conception de telles exploitations dont les taux de défruitement peuvent atteindre $55 \%$ est soumise à deux règles fondamentales :

- les piliers doivent être stables pour une durée "infinie »;

- la largeur des bandes ne doit pas excéder une valeur critique au-delà de laquelle l'équilibre global du massif se modifie radicalement.

De telles exploitations tendent à se développer dans des conditions de plus en plus diverses et nécessitent une connaissance accrue du phénomène.

Nous proposons, dans cet article, une méthode de prévision de l'affaissement en exploitation partielle. Cette méthode repose sur une étude statistique de différentes expériences d'exploitation partielle dans des mines de charbon, en France, au Royaume Uni, en Pologne et en Australie et tient compte de l'environnement géologique et minier de l'exploitation.

\section{Rappel des connaissances actuelles}

Une exploitation minière a deux sortes d'effets sur les terrains qui l'entourent: des effets transitoires qui suivent la progression du chantier et des effets définitifs qui resteront pratiquement non modifiés après l'arrêt du chantier. Ces effets se ressentent à la surface et y déterminent des déplacements du sol (fig. 1). Le déplacement vertical est l'affaissement proprement dit. Les mouvements différentiels associés sont la pente pour l'affaissement et la déformation pour le déplacement horizontal. Ces mouvements différentiels sont les principaux responsables des dégâts aux constructions et doivent donc faire l'objet de prévisions les plus sûres possibles.

Nous nous intéresserons, dans ce qui suit, aux mouvements finals sur lesquels repose toute étude prévisionnelle. Dans ce cas, on peut relier assez précisément la valeur de la déformation maximale dm ou de la pente maximale pm sur un site à l'affaissement maximal Am avec une relation du type

$$
\mathrm{dm}=\mathrm{k}_{1} \frac{\mathrm{Am}}{\mathrm{P}} ; \quad \mathrm{pm}=\mathrm{k}_{2} \frac{\mathrm{Am}}{\mathrm{P}}
$$

$\mathrm{dm}$, déformation maximale,

pm, pente maximale,

Am, affaissement maximal,

$P$, profondeur.

$k_{1}, k_{2}$ coefficients à déterminer en fonction de la géométrie de l'exploitation et du type de terrain en surface.

Les exploitations partielles par bandes et piliers ont des longueurs de chassage grandes par rapport à la largeur de la bande (plus de 5 fois supérieures en général), de sorte que l'on peut considérer que les phénomènes se produisent dans des plans verticaux comme sur la figure 2. Les caractéristiques géométriques de l'exploitation se réduisent à :

- la profondeur $P$ et l'ouverture $O$ de la couche exploitée;

- les largeurs W du pilier, L de la bande et LT du panneau (largeur totale du chantier).

Deux méthodes ont été proposées pour prévoir l'affaissement maximal Am, sur un site, à partir de ces caractéristiques géométriques :

- ORCHAD [1] (National Coal Board, NCB) propose de faire la somme des profils (fig. 3) d'affaissements relatifs aux différentes bandes et de mesurer l'affaissement maximal sur le profil ainsi obtenu. 


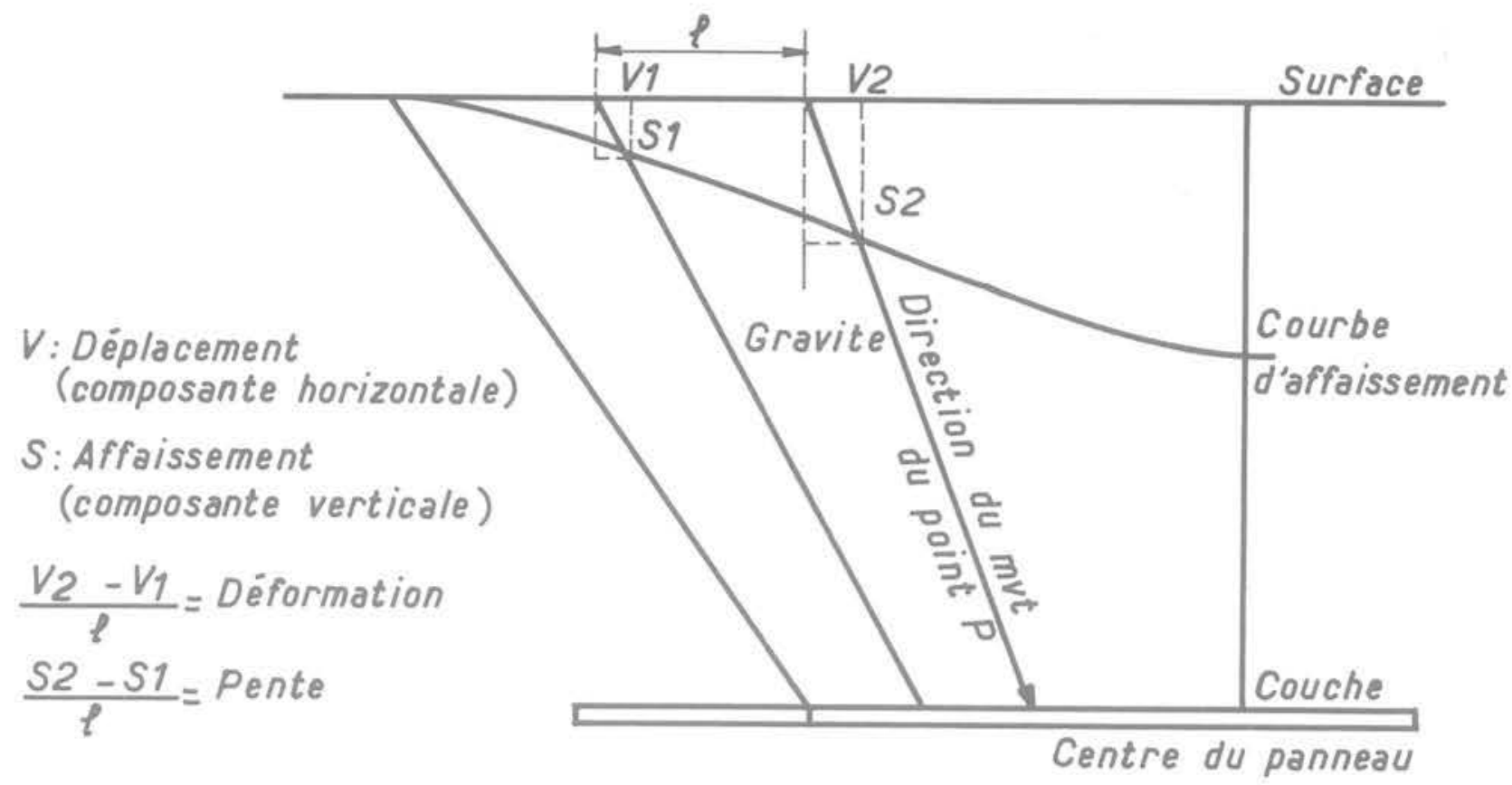

Fig. 1 Relation affaissement/déplacement

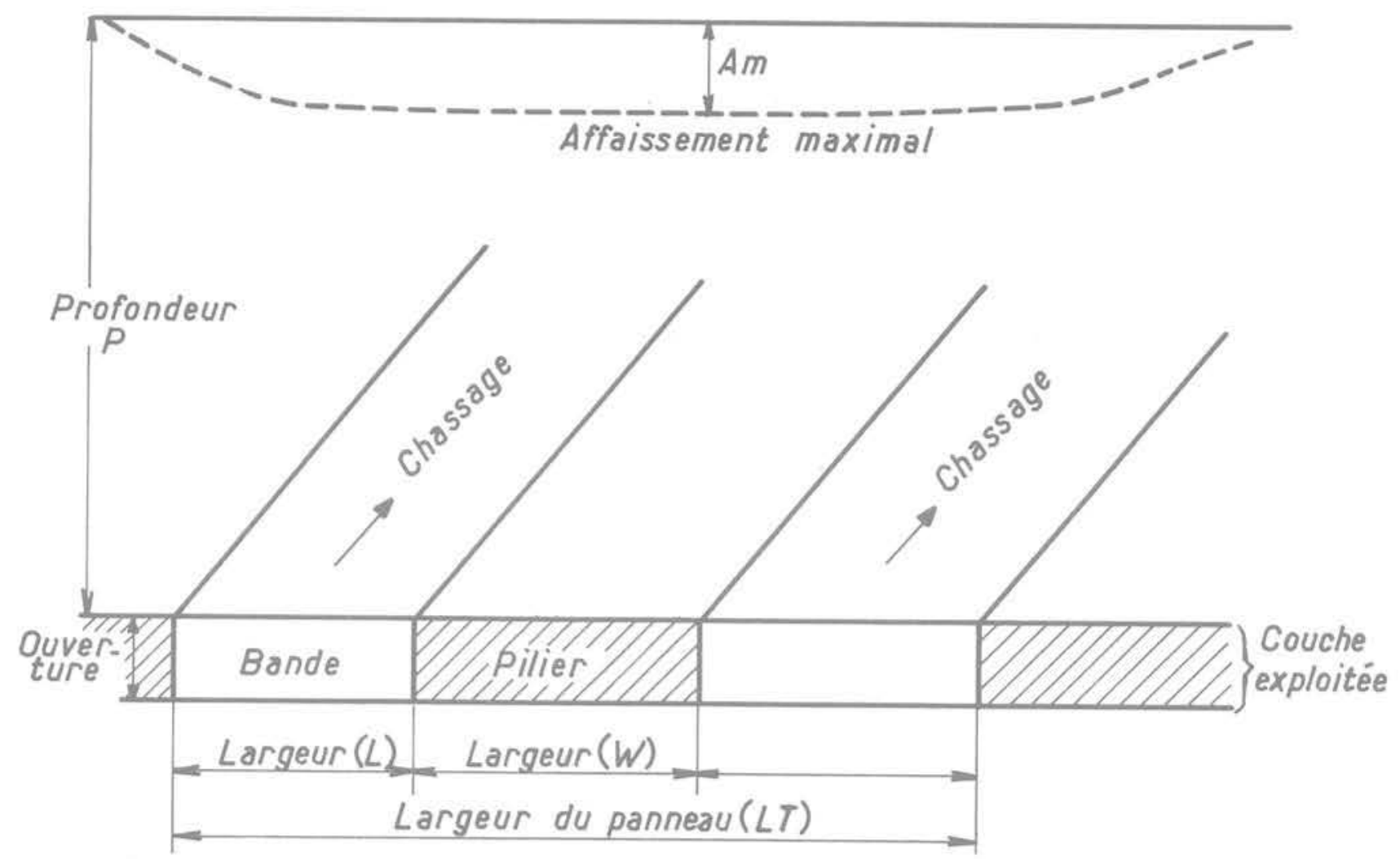

Fig. 2 Les caractéristiques géométriques des exploitations partielles

Sur un certain nombre de cas(") d'exploitation au Royaume Uni, cette méthode permet de retrouver à $10 \%$ près les affaissements mesurés.

- WARDELL [2] $\left({ }^{* *}\right)$ analyse un modèle de mouvements du massif, qui tient compte du comportement du pilier et du vide causé par l'exploitation et propose d'évaluer l'affaissement maximum Am en fonction de la charge imposée aux piliers $\mathrm{Ca}$, de l'élancement des piliers $\mathrm{O} / \mathrm{W}$, et du volume du vide

(") Ces cas ont été repris dans l'étude qui suit.

(“) Bureau d'ingènieurs consultants : K. Wardell and Partners.
$\mathrm{L} \times \mathrm{O}$ selon l'expression :

$$
\mathrm{Ca} \times \frac{\mathrm{O}}{\mathrm{W}} \times \mathrm{L} \times 0
$$

d'où il tire: $\frac{A m}{0}=f\left(C a \times \frac{O}{W} \times L\right)$.

La fonctionnelle f est établie à partir de 10 cas; elle est représentée sur la figure 4 .

La première méthode fait l'hypothèse d'additivité des effets de chacune des bandes à la surface, hypothèse difficile à justifier "a priori" suivant la nature des terrains. L'extrapolation est toujours délicate avec la seconde méthode dont la fonctionnelle n'est définie 


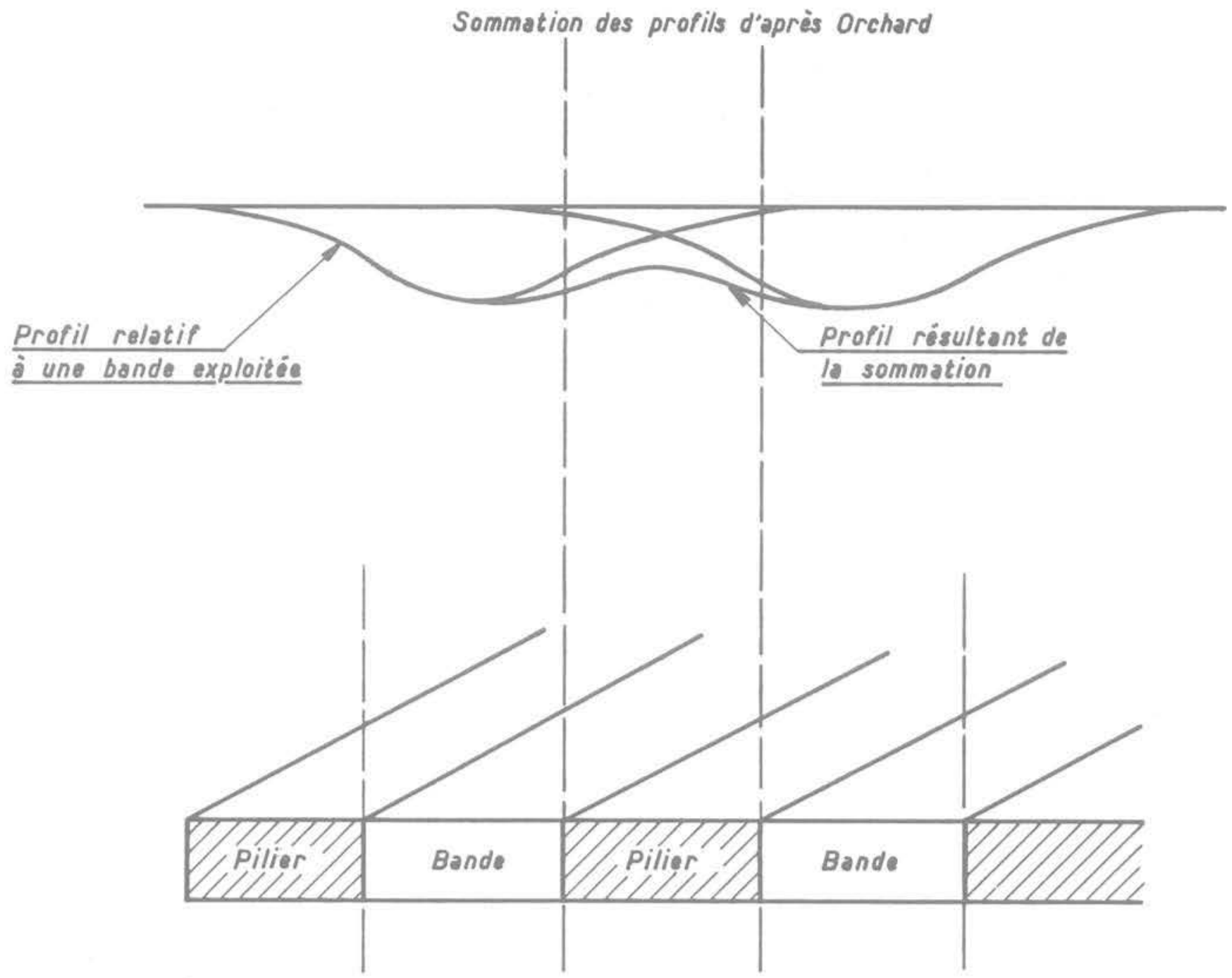

Fig. 3 Schéma des méthodes d'exploitation partielle par bandes et piliers

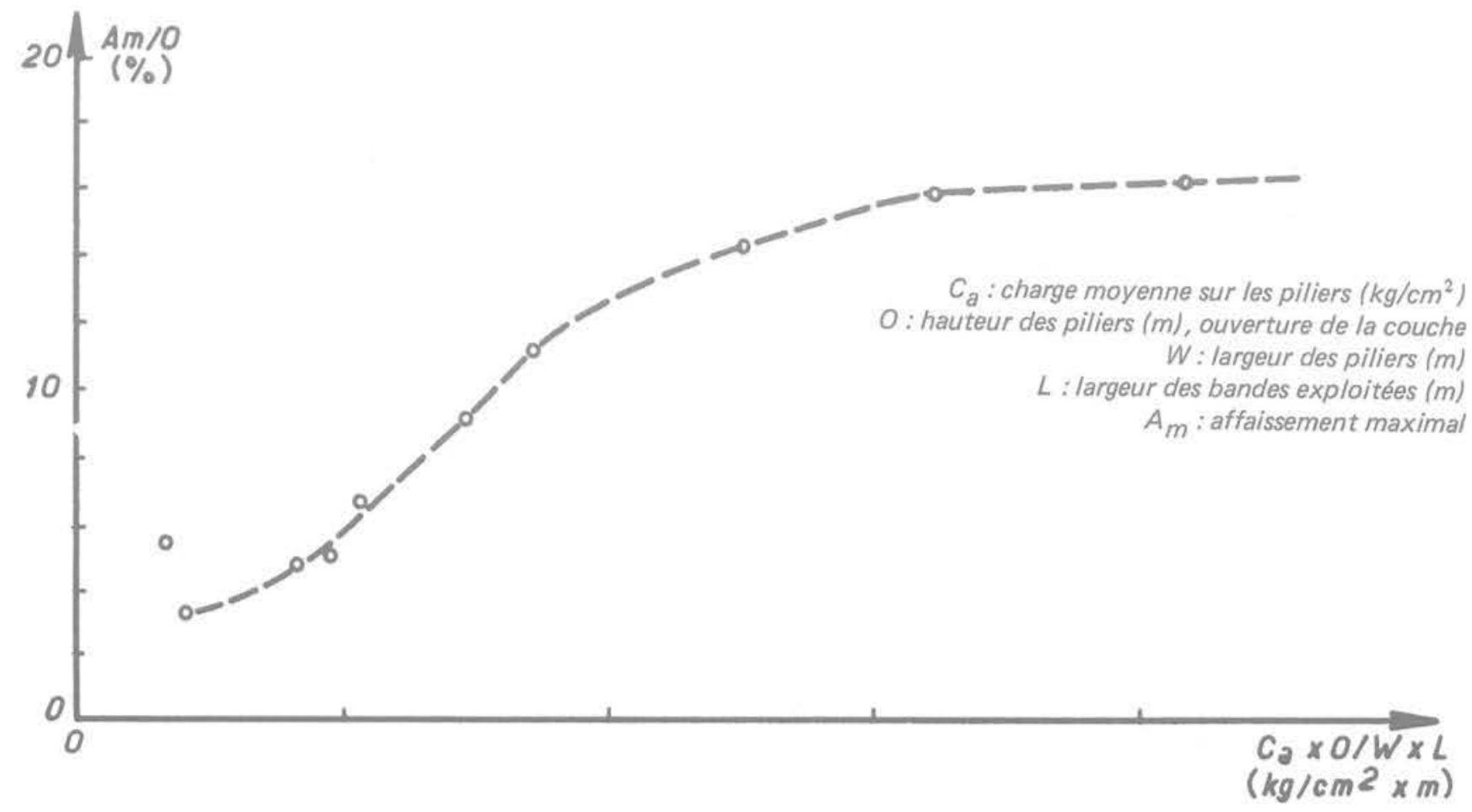

Fig. 4 Affaissement du A des méthodes de dépilage partiel par bandes et piliers (d'après Wardell et Eynon) 
que pour 10 cas d'environnement géographique et probablement géologique voisins.

Ces méthodes ne faisant intervenir que les seules caractéristiques géométriques de l'exploitation, nous avons voulu étudier par des méthodes statistiques, l'influence de facteurs naturels et tenter de préciser le mécanisme du comportement des terrains au-dessus de ce type d'exploitation.

\section{Collecte et traitement des données}

Le traitement statistique qui a été réalisé a porté sur des observations faites dans des bassins houillers divers [4]:

4 cas ont été étudiés en France : 3 dans les houillères du Bassin du Nord et du Pas-de-Calais et un dans les houillères d'Aquitaine;

17 cas ont été rassemblés par le National Coal Board provenant de bassins aussi différents que ceux des Midlands ou d'Écosse;

7 cas ont èté étudiés en Australie, dans les Northern Coalfields:

3 cas ont été rassemblés dans la région de Katowice, dans les charbonnages polonais.

Pour réaliser cette étude statistique, nous avons caractérisé l'environnement géologique et minier de l'exploitation par les éléments suivants

- la présence d'exploitations antérieures et le remblayage;

- la résistance globale du massif (RE) partagée en trois classes définies par la proportion de bancs très résistants (grès par exemple) dans le recouvrement.

- Recouvrement " compétent (*) (REC) : 50 à $80 \%$.

- Recouvrement “intermédiaire" (REI) : 20 à $50 \%$.

- Recouvrement "plastique " (REP) : moins de $20 \%$.

- la distance séparant la couche du haut-toit qui a été définie comme le premier banc résistant pouvant supporter la flexion. Trois classes ont été constituées:

- Haut toit "bas* (HTb) : à moins de $20 \mathrm{~m}$ de la couche.

- Haut toit "moyen " [HTM] : entre 20 m et 30 m de la couche;

- Haut toit "haut" (HTh) : à plus de $30 \mathrm{~m}$.

- le pendage (supérieur ou inférieur à $35^{\circ}$ ) et la tectonique (souple ou rigide).

Un paramètre "qualité de la mesure " a été introduit pour tenir compte du fait que dans certains cas, les affaissements avaient été sous-estimés en raison de la durée trop courte des mesures, ou au contraire surestimés en raison de l'influence d'anciennes exploitations non stabilisées.

Dans l'échantillon ainsi rassemblé, les affaissements mesurés varient entre 1 et $60 \mathrm{~cm}$, les ouvertures se répartissent entre 2 et $7 \mathrm{~m}$ à des profondeurs de 100 à $900 \mathrm{~m}$

\subsection{Le traitement}

Le traitement a été conduit avec deux techniques d'analyse très différentes

- I'analyse en composantes principales [3];

- la régression multiple linéaire.

(*) Compétent : néologisme désignant un massit constitué en grande partie de bancs épais et résistants. Une définition plus précise sera donnée dans le texte.
L'analyse en composantes principales est une technique de description et de classification des observations qui permet de faire apparaître simplement certaines structures caractérisant l'ensemble des données. Chaque observation peut être représentée par un point dans un espace comportant autant de dimensions que de variables : chacune des variables occupe un axe de coordonnées. L'échantillon étudié est alors représenté par un " nuage n de points qui a la forme d'un ellipsoîde. L'analyse en composantes principales consiste à projeter ce nuage de points sur les axes principaux de cet ellipsoïde : le plus grand axe est appelé axe 1, et fait apparaître des différenciations caractéristiques à l'intérieur de l'échantillon; la figure 5 , qui représente cette projection du nuage sur cet axe, montre l'hétérogénéité entre les cas australiens et les cas européens. On peut également projeter le nuage de points sur le plan constitué par deux axes principaux de l'ellipsoïde, comme sur la figure 6 . Sur cette figure, on voit apparaitre une partition nette entre les différents bassins européens. On verra que cette différenciation géographique pourra être interprétée en terme de différences géologiques.

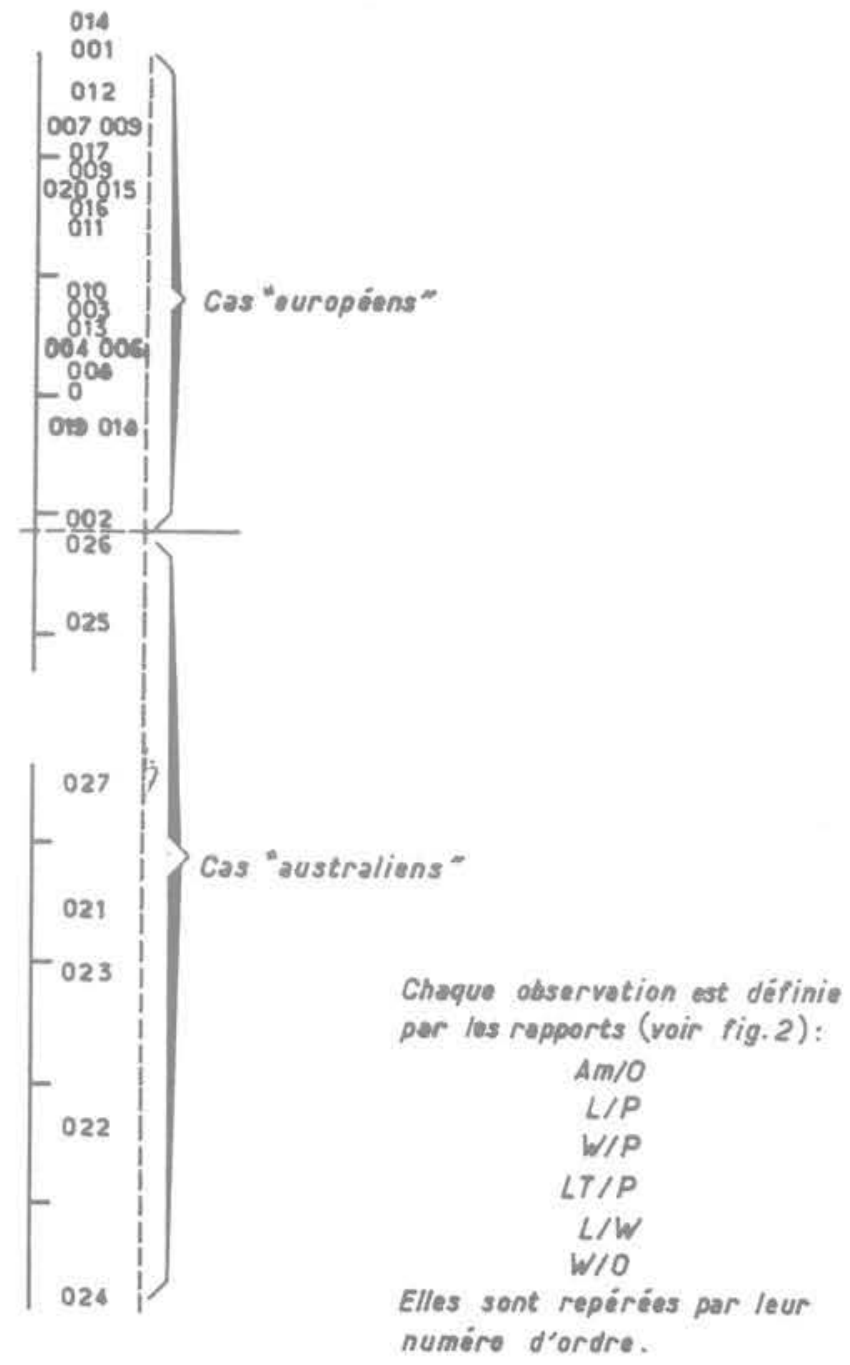

Fig. 5 Projection des différents individus sur l'axe 1

(analyse dans l'espace des variables) 


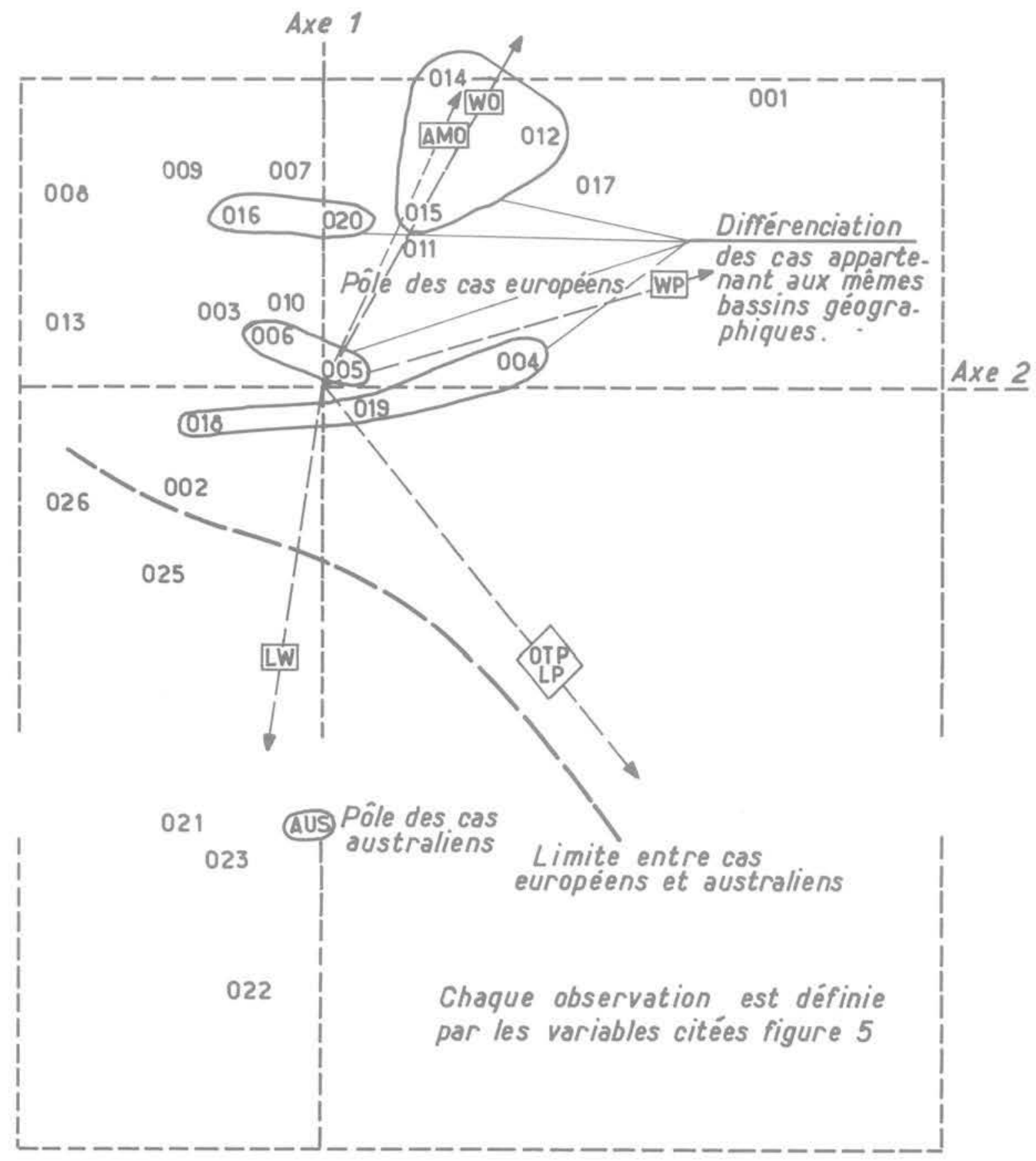

Fig. 6 Projection des individus sur le premier plan principal 
La régression multiple est une technique qui permet une approche plus quantitative; elle fait, toutefois, un certain nombre d'hypothèses :

La première hypothèse est celle de l'additivité de l'infıuence des différentes variables:

On admet que l'on peut écrire :

$$
A m=\sum_{i=1}^{i=n} x i \mathrm{Vi}
$$

avec Am : affaissement maximal:

$\mathrm{Vi}$; ième variable explicative:

$x i$ : coefficient recherché.

L'analyse physique du phénomène, que ce soit au niveau du toit, des piliers chargés ou du recouvrement, conduit à penser que les variables quantitatives sont multiplicatives. L'analyse sera donc conduite sur le logarithme des variables.

Cette hypothèse de travail est confirmée par les approches bibliographiques.

Les variables qualitatives (recouvrement, hauteur de toit, qualité de la mesure) sont suggérées par l'analyse en composantes principales (influence de l'environnement géologique).

La seconde hypothèse fondamentale de la technique de régression est celle de l'indépendance de l'influence des diffèrentes variables.

Afin d'éviter les erreurs d'interprétation dues éventuellement aux hypothèses de la régression, chaque analyse a été conduite en trois temps:

- sur le groupe des cas "européens" (groupe 1);

- sur le groupe des cas "australiens" (groupe 2):

- sur l'ensemble des cas.

a) La première analyse présentée ici est faite sur les variables quantitatives explicatives suivantes:

- ouverture $\mathrm{O}$;

- profondeur $\mathrm{P}$.

- largeur de bande Li

- largeur du panneau LT:

- rapport largeur de bande sur largeur de pilier $\frac{L}{W}$ :

L'affaissement maximal est la variable expliquée.

Les résultats du calcul cités sur les tableaux 1, 2 et 3 , permettent de tirer les conclusions ci-après :

- avec un coefficient de corrélation multiple supérieur à $0,9(0,924)$, la régression explique plus de $80 \%$ des variations de l'affaissement. Les variables significatives sont alors l'ouverture, la largeur de bande et la classe de recouvrement pour le premier groupe.

Les résultats de la régression s'écrivent alors :

$$
\log A m=1,07 \log 0+0,8 \log L-2,6
$$

dans le cas de recouvrement "plastique" (REP) et

$$
\log A m=1,07 \log 0+0,8 \log L-(2,6+0,29)
$$

dans le cas de recouvrement "intermédiaire " (REI).

En prenant I'exponentielle de chacun des membres, on obtient :

$$
\begin{aligned}
& A m=2,5 \cdot 10^{-3} \times 0^{1.07} \times L^{0.8} \text { (REP) } \\
& A m=1,3 \cdot 10^{-3} \times 0^{1,07} \times L^{0.8} \text { (REI). }
\end{aligned}
$$

Ouverture de la couche et largeur de la bande ont un exposant voisin de 1 ; à dimensionnement constant, l'affaissement maximal est plus fort dans le cas de recouvrement plastique, ce qui paraît physiquement réaliste.

- avec un coefficient de corrélation plus faible de
0,901 , les variables significatives du deuxième groupe sont :

- la largeur de bande (exposant voisin de 1);

- la classe de hauteur de toit.

- le coefficient de corrélation diminue légèrement $(0,886)$ quand l'ensemble des cas sont réunis pour l'analyse. L'ouverture et la largeur de bande demeurent des variables significatives avec un exposant voisin de 1.

L'ensemble des cas se divise en deux groupes suivant que le recouvrement est "plastique " ou non.

Enfin, l'affaissement ne dépénd, dans l'échantillon étudié, ni de la largeur de pilier, ni du rapport largeur de bande sur largeur de pilier qui est lié directement à la charge sur les piliers.

Avant de commenter plus avant et d'utiliser les résultats de ces calculs de régression, il est nécessaire d'en étudier la "stabilité ", c'est-à-dire de vérifier qu'ils ne sont pas modifiés de manière importante par une petite modification de l'échantillon ou des variables étudiés.

Pour cela, les calculs suivants ont été effectués également :

- sans le cas Cagnac (Houillères d'Aquitaine), d'ouverture très forte, on obtient des résultats équivalents;

- sans les variables qualitatives décrivant la composition du toit, "ouverture " et "largeur de bande" restent les principales variables significatives. La prise en compte de ces dernières améliore nettement la qualité de la corrélation.

b) Une deuxième analyse a été effectuée, pour mieux apprécier le rôle des différents facteurs. Elle a consisté à prendre le rapport $\mathrm{Am} / \mathrm{O}$, suggéré par l'analyse précédente, comme variable expliquée.

Les résultats obtenus sont équivalents aux précédents: le coefficient de corrélation multiple est légèrement amélioré sur l'analyse de l'ensemble des observations $(0,896)$.

Les résultats de cette analyse sont illustrés sur la figure 7. Cette figure montre que tous les cas se répartissent autour de deux droites dans le plan défini par les paramètres: largeur de bande et rapport affaissement maximal sur ouverture. Cette partition définit des exploitations de type "compétent" (cidessus "compétent" et " intermédiaire") et des exploitations de type "plastique".

Les droites de corrélation simple issues de cette partition sont parallèles, ce qui indique que les mécanismes d'affaissement sont équivalents dans les deux cas.

c) L'absence d'influence des paramètres géométriques qui paraissent a priori importants dans le dimensionnement d'une exploitation partielle, comme la largeur des piliers et la profondeur de l'exploitation s'explique par les particularités de l'ensemble des cas qui ont été analysés :

- d'une manière générale, les piliers et les bandes ont été dimensionnés de manière à limiter les affaissements à la surface à quelques centimètres ou quelques décimètres, pour empêcher les dégâts aux structures existantes. Le domaine des variations de l'affaissement mesuré est donc faible, et l'effet des facteurs qui tendent normalement à augmenter son amplitude comme par exemple la charge sur les piliers, a pu être "corrigé" au moment de la conception de l'exploitation par la 


\begin{tabular}{|c|c|c|c|c|}
\hline \multicolumn{2}{|c|}{$\begin{array}{l}\text { Coefficient de corrélation } \\
\text { Terme constant }\end{array}$} & \multicolumn{3}{|c|}{$\begin{array}{c}0,924 \\
-2,5883\end{array}$} \\
\hline Variable & Coefficient & $\begin{array}{l}\text { Ecart-type de } \\
\text { ce coefficient }\end{array}$ & $\begin{array}{c}\text { Valeur du } T \text { de } \\
\text { Student }\end{array}$ & $\begin{array}{l}\text { Coefficient de } \\
\text { cor:partielle }\end{array}$ \\
\hline 0 & 1,0737 & 0,1593 & 6,7419 & 0,8531 \\
\hline$L$ & 0,7963 & 0,1148 & 6,9362 & 0.8596 \\
\hline$R E I$ & $-0,2871$ & 0,0703 & $-4,0822$ & 0,7036 \\
\hline
\end{tabular}

Tableau 2 Groupe 2

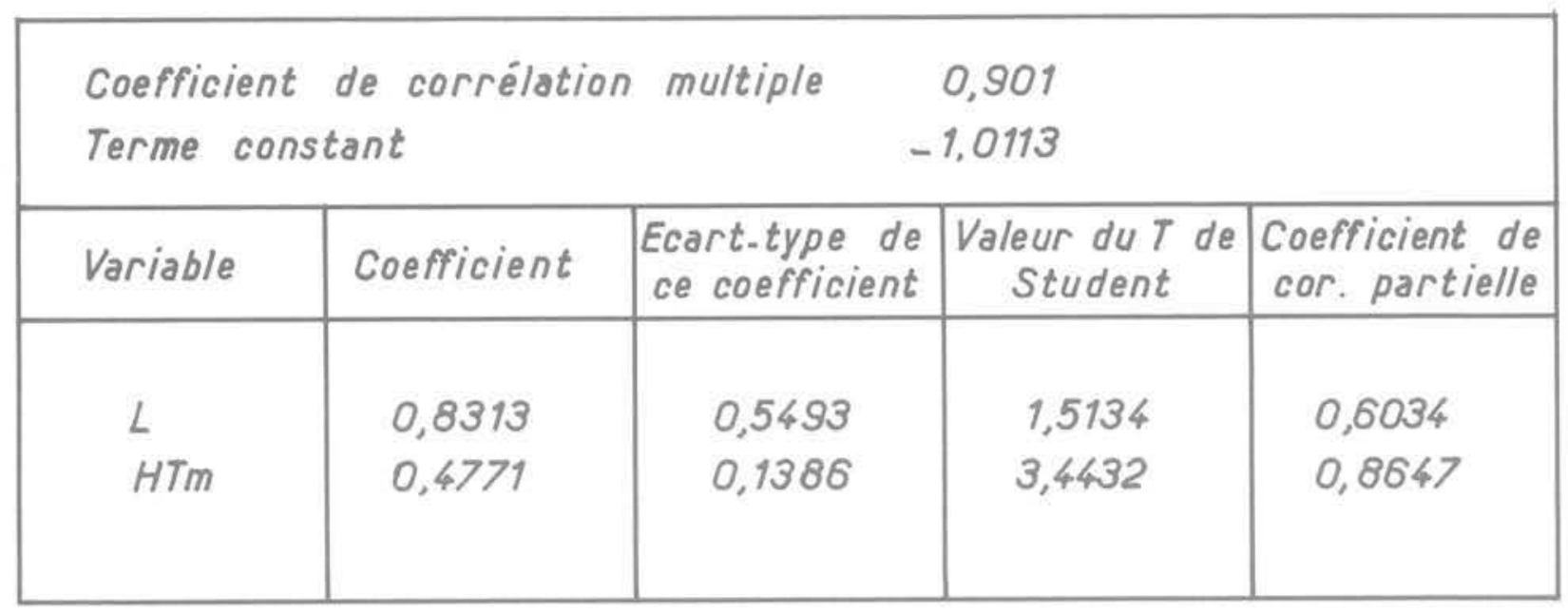

Tableau 3 Groupe 3

\begin{tabular}{|c|c|c|c|c|}
\hline \multicolumn{2}{|c|}{$\begin{array}{l}\text { Coefficient de corrélation } \\
\text { Terme constant }\end{array}$} & \multicolumn{3}{|c|}{$\begin{array}{c}0,886 \\
-3,4765\end{array}$} \\
\hline Variable & Coefficient & $\begin{array}{l}\text { Ecart-type de } \\
\text { ce coefficient }\end{array}$ & $\begin{array}{l}\text { Valeur duT de } \\
\text { Student }\end{array}$ & $\begin{array}{l}\text { Coefficient de } \\
\text { cor.partielle }\end{array}$ \\
\hline 0 & 1,0043 & 0,2292 & 4,3811 & 0,6745 \\
\hline$L$ & 0,9684 & 0,1509 & 6,4173 & 0,8010 \\
\hline HTm & 0,3176 & 0,0875 & 3,6299 & 0,6035 \\
\hline$R E P$ & 0,6630 & 0,0886 & 7,4875 & 0,8421 \\
\hline
\end{tabular}



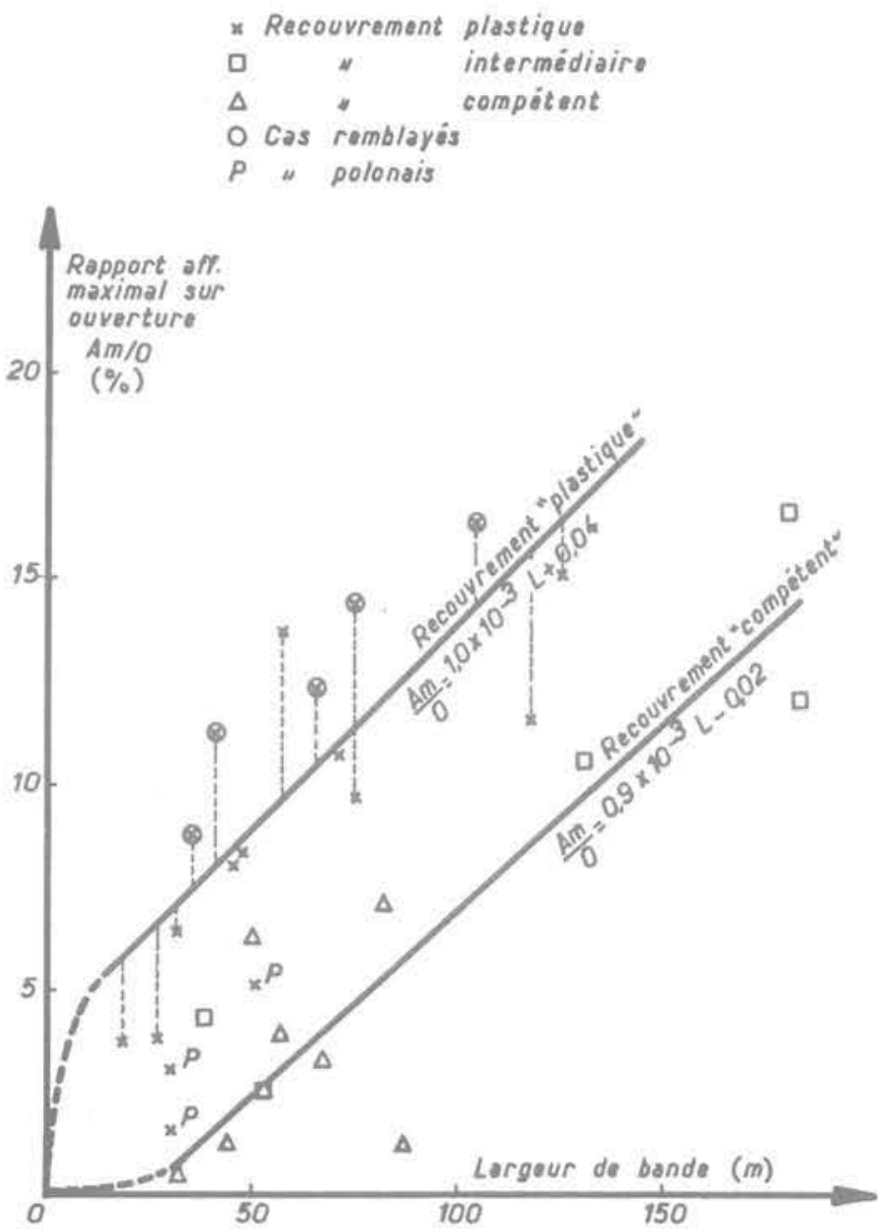

Fig. 7 Evolution de l'affaissement maximal $A_{m}$ en fonction de la longueur des bandes. Différenciation des classes de recouvrement

modification d'un autre facteur (par exemple l'augmentation de la largeur des piliers).

- le dimensionnement des exploitations a été fait. pour la plupart des cas étudiés, selon des règles analogues, qui font que la largeur des piliers est systématiquement voisine de la largeur des bandes exploitées : l'étude de la corrélation entre la largeur des piliers et les autres variables (largeur de bande, profondeur, ouverture, taux de charge sur les piliers) montre que $80 \%$ des variations de la largeur des piliers peuvent être expliqués par la variation de la largeur des bandes exploitées. La largeur des piliers augmente significativement avec la profondeur, de même que la largeur des bandes exploitées. Ainsi, les variations de l'affaissement avec la largeur des piliers ou la profondeur sont prises en compte par la liaison qui existe entre la largeur des bandes et ces paramètres.

Enfin, il est intéressant de noter que dans le cas des exploitations partielles, le remblayage des chambres ne semble pas avoir d'influence directe sur l'affaissement : sur la figure 7, les exploitations remblayées sont homogènes aux exploitations foudroyées et leurs points représentatifs sont situés au-dessus de la courbe supérieure. Mais le remblayage garde un rôle dans la stabilité des piliers notamment en assurant un frettage quasi immédiat des piliers. On peut dire que par ce biais, il a bien pour rôle de limiter les affaissements.
Rapport entre affaissement

au temps $t$ et affaissement

maximum rinal.

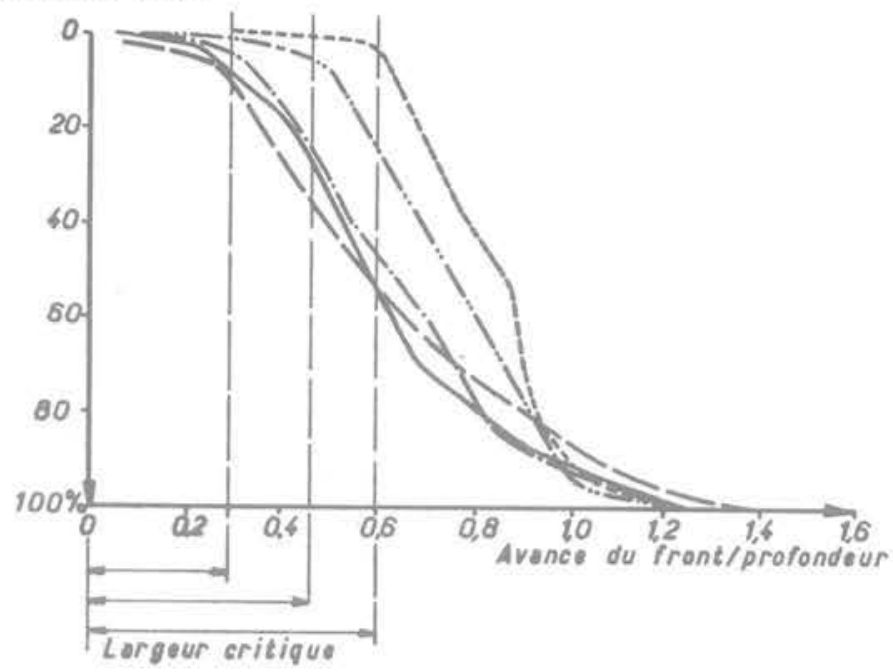

Lithographio

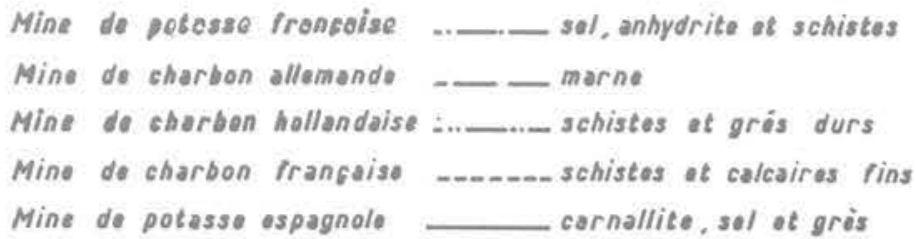

Fig. 8 Comparaison des différentes courbes d'affaissement en fonction de l'avance du front. Définition de la largeur critique (courbes citées par Wardell et Eynon)

\section{2 Éléments pour une méthode de prévision des affaissements en exploitation partielle}

Le dimensionnement d'une exploitation partielle consiste à choisir la largeur des piliers stables, la largeur des bandes et la longueur de chassage des chantiers de manière à ce que l'affaissement maximal attendu reste inférieur à une quantité définie en fonction de la protection de la surface qui est nécessaire.

Dans le cas que nous avons étudié, la longueur de chassage des chantiers est sans influence sur les affaissements de la surface : ce paramètre est choisi seulement en fonction de la planification générale des travaux.

Le choix de la largeur du pilier et de la largeur de bande dépend du matériau exploité et de son environnement.

De nombreuses méthodes, liées à des "ẻcoles" différentes, permettent de calculer la largeur du pilier stable. Une analyse critique dépasserait le cadre de cet article. Notons toutefois que la détermination de cette largeur est une étape clé dans le dimensionnement d'une exploitation partielle. Un calcul optimiste, en effet, pourrait conduire à une rupture des piliers et par conséquent à un affaissement souvent catastrophique de la surface. Un calcul pessimiste, par contre, peut aboutir à une largeur de pilier anormalement grande. 


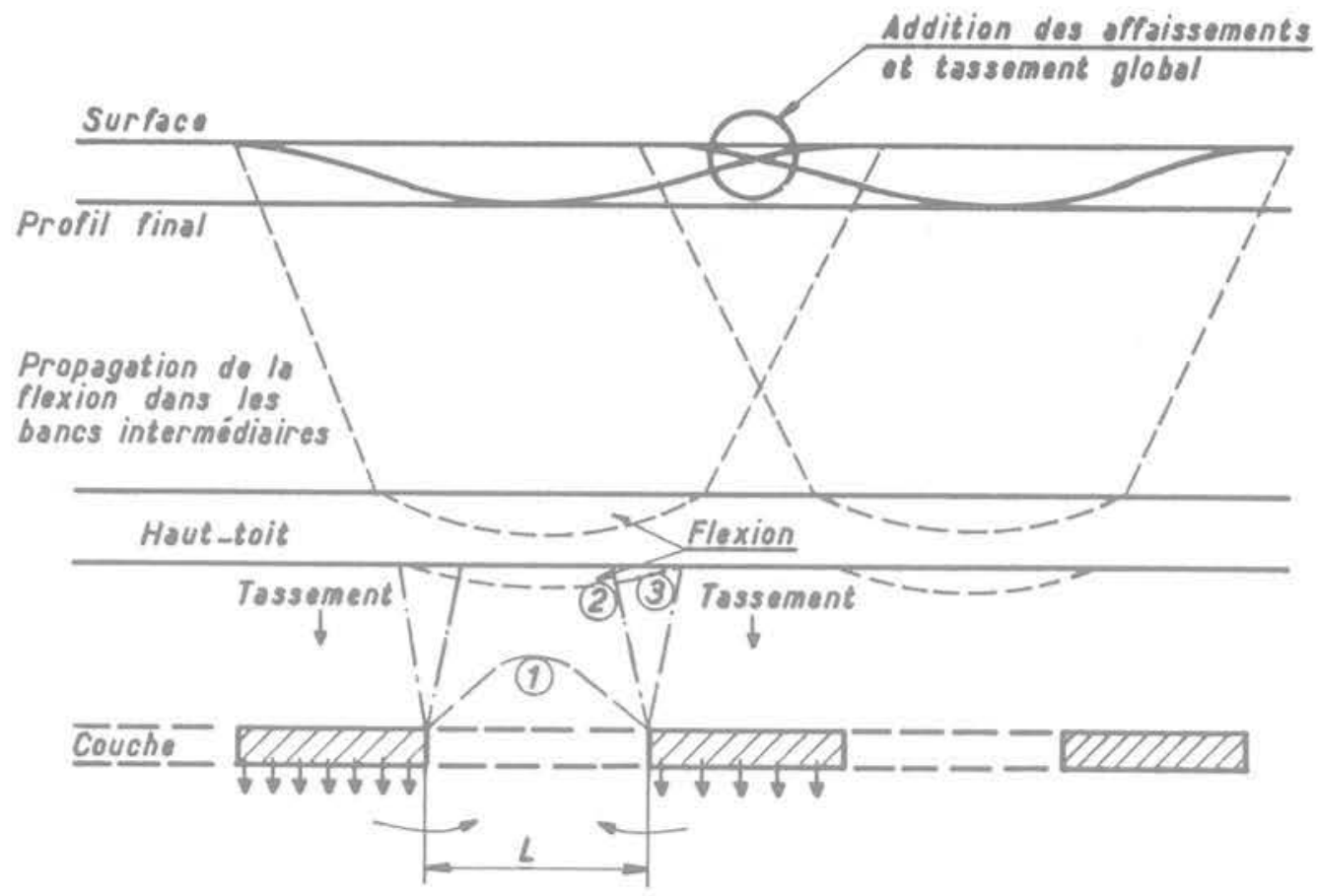

\begin{abstract}
Apres l'ouverture de la chambre (1) , lo voûte se déplace en (2) Les terrains prennent ensuite l'âtat d'équilibre en (3)

jusqu'au haut-toit qui flëchit.
\end{abstract}

Fig. 9 Terrains plastiques

Dans ce cas, la cuvette d'affaissement ne présente plus un fond plat sur sa plus grande partie, mais des ondulations. Ces ondulations peuvent entrainer des déformations horizontales anormalement fortes, qui font perdre une grande partie du bénéfice de l'exploitation partielle.

La largeur de la bande doit être déterminée de façon à conserver l'intégrité du haut-toit. Les mesures à la surface permettent, sur un site, de définir la largeur critique au-delà de laquelle le massif change de comportement global à cause de la rupture du banc porteur principal. Sur les courbes de la figure 8 définies par l'affaissement en fonction de l'avance du front pour des chantiers très larges, on peut distinguer trois parties :

- une phase en plateau au cours de laquelle I'affaissement évolue lentement;

- une phase d'accélération des déplacements;

- une phase de ralentissement tendant vers un équilibre.

Dans ces chantiers, la longueur de chassage est équivalente à la largeur de bande dans les exploitations partielles. L'équilibre initial du massif est conservé si on reste dans la phase en plateau; la largeur du chantier correspondant constitue la largeur critique. A titre d'exemple, on peut considérer que dans le cas de recouvrement "compétent", cette distance peut atteindre $45 \%$ de la profondeur.

Pour apprécier le rôle de la largeur de bande et la définition du type de recouvrement, points clés d'une étude d'exploitation partielle, nous proposons un modèle simple de mécanisme du massif.

Dans le cas de terrains de type "plastique " comme illustré sur la figure 9 , il y a enfoncement et tassement des piliers dans les terrains du mur et du toit, qui se tassent également. Au-dessus du vide, un foudroyage partiel se produit avec foisonnement des terrains et l'ensemble des bancs compris entre le haut toit et les piliers se tassent en raison du surcroît de charge. Le haut toit fléchit mais repose sur les terrains foisonnés et ne se détruit pas, préservant l'équilibre global du massif. La flexion de ces bancs se propage jusqu'au jour. Globalement, pour l'ensemble du panneau, on obtient en surface une cuvette à fond plat en raison du tassement général de l'ensemble et de la répartition de la flexion du haut toit dans le massif. La largeur de la bande croissant, le supplément de charge sur les piliers et la flexion du haut toit augmentent ainsi que l'affaissement. Dans ce cas, un affaissement se produit même pour de petites longueurs exploitées.

Pour les terrains de type "compétent" (fig. 10), le comportement du massif présente un seuil. Pour de faibles largeurs de bande, la rupture des bancs n'atteint pas le haut-toit et, par conséquent, on n'enregistre en surface qu'un affaissement minime dû au tassement et à l'enfoncement des piliers. Quand le seuil est dépassé, c'est-à-dire quand la rupture des bancs atteint le haut toit, il y a flexion du haut-toit et affaissement en surface. Dans ce cas toutefois, l'affaissement est plus faible que dans le cas précédent car les terrains étant plus raides, la flexion des bancs et les tassements globaux sont réduits.

Cette approche du mécanisme des mouvements du massif et de la surface permet de mieux guider le choix de l'exploitation pour un type de recouvrement donné. Une fois ce choix fait, il reste à se placer sur l'abaque de la figure 7 pour déterminer l'affaissement maximal à prévoir. 


\section{Haut-toit}

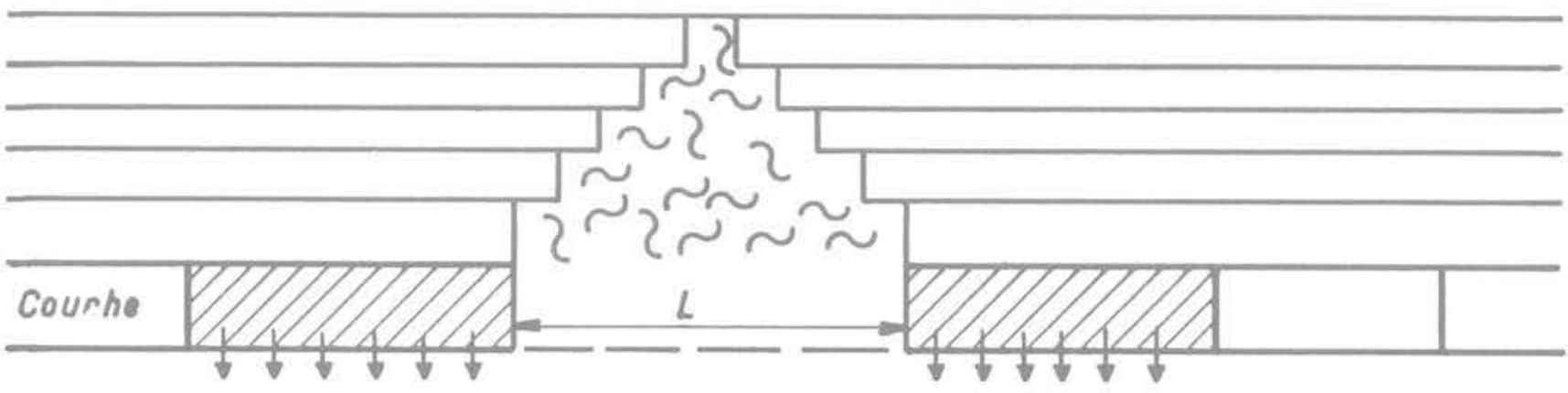

Fig. 10 Terrains épais et résistants (avant le seuil)

\section{Conclusion}

A partir de l'étude statistique de différents cas d'exploitation partielle nous proposons une méthode de prévision de l'affaissement maximal et corrélativement un modèle pour le mécanisme des mouvements dans le massif.

Ce modèle est en accord avec la méthode proposée par ORCHard. Sans qu'il y ait simplement additivité des effets des différentes bandes, on a pu constater que la sommation des profils rendait compte dans une certaine mesure de la réalité. Les prévisions à partir de cette méthode sont en général comprises entre les deux droites définies sur la figure 7 .

Le modèle de comportement de WARDELL, défini à partir du volume du vide exploité, le paramètre LO. produit de la largeur de la bande par l'ouverture, est aussi en accord avec la méthode proposée dans cet article.

Toutefois, le modèle présenté ici tient compte de la nature des terrains environnant l'exploitation et permet donc une extrapolation raisonnable à des cas différents.

II serait, en particulier intéressant de confronter ce modèle avec les mesures d'affaissement effectuées lors d'exploitations partielles dans d'autres matériaux, comme le fer ou la potasse.

\section{Références bibliographiques}

R. J. Orchard - "Longwall Partial Extraction Systems. " Mining Engineer CXXIX 1969-1970.

K. Wardell - "Structural Concept of Strata Control, n Mining Engineer CXXVII 1967-1968.

Caillez et Pages - "Introduction à l'Analyse des données." Smash éditeur.

J. Arcamone - "Méthodologie de prévision des affaissements miniers en exploitation totale et partielle. " Thèse INPL juin 1980. 